\title{
Rapid Bicycle Gear Switching Based on Physiological Cues
}

\author{
Edward Hallett, Richard Woodward, Simon Schultz, and Ravi Vaidyanathan
}

\begin{abstract}
This paper discusses the merits of Mechanomyography (MMG) sensors in capturing and isolating muscle activity in high interference environs, with application to 'hands free' gear shifting on a bicycle for users with limited extremity movement. MMG (acoustic) muscle sensing provides a simple and rugged alternative to physiological sensing for machine interface in the field, but suffers from interfering artifacts (in particular motion) which has limited its mainstream use. We introduce a system fusing MMG with a filter based on Inertial Measurement (IMU) to isolate muscle activity in the presence of interfering motion and vibrations. The system identifies user-initiated muscle trigger profiles during laboratory testing, allowing parameterization of MMG and IMU signals to identify purposeful muscle contractions (triggers) and to omit false triggers resulting from cycle/road vibration or rider movement. During laboratory testing the success rate of trigger identification was $88.5 \%$ while cycling with an average of 0.87 false triggers $/ \mathrm{min}$. During road testing the success rate was 72.5\% and false triggers were more frequent at $3.7 / \mathrm{min}$. These results hold strong promise for alternative triggering mechanisms to the standard bar-end shifters used in current offthe-shelf cycling group sets, enabling amputees or people of reduced arm or hand dexterity to change gears while riding. Further testing will explore the use of signal filters on MMG data and further use of IMU data as feedback to increase false triggers rejection. Wider applications include a broad range of machineinteraction research.
\end{abstract}

Index Terms - Bicep Contractions, Butterworth Filter, Electro-Mechanical Coupling, Human Machine Interface, Inertial Measurement Unit (IMU), Mechanomyogram (MMG), Noise Reduction.

\section{INTRODUCTION}

A bicycle bio-signal gear system would grant arm amputees or individuals with reduced hand dexterity the ability to change gears without additional arm/hand movement. Those with impeded hand or arm use have had little opportunity to be involved in the sport, with no current road cycling events in parasports for arm amputees in events such as the coming 2016 Paralympics in Rio [1]. These events are limited to wheelchair or arm-pedaling. The main objective of this project is to explore the use of MMG and IMU sensors to instigate a gear change on a bicycle as a means of supporting activity and independence for patients with upper extremity impairment.

Manuscript submitted March 15, 2015. This work was supported in part by the Mechanical Engineering Dept. Imperial College London, and the Rio Tinto Sports Innovation Challenge (RTSIC) project.

E. Hallett, R. Vaidyanathan and Richard Woodward are with the Dept. of Mechanical Engineering, Imperial College London, England, SW7 2AZ UK (email:r.vaidyanathan@imperial.ac.uk, richard.woodward09@imperial.ac.uk).

Simon Schultz is with the Dept. of Bioengineering, Imperial College London, England, SW7 2AZ UK (email: s.schultz@imperial.ac.uk).

\section{A. Sensing Modality}

Research in smoother and more natural Human-Machine Interfaces (HMIs) for assistive technology has proliferated in recent years; body-worn devices such as exoskeletons, instrumented gloves, inertial or electromagnetic motion tracking sensors, Electroencephalographic (EEG) brain activity sensors, Electromyographic (EMG) and MMG muscular activity sensors are all commonplace. Performance in harsh environs for activities such as cycling, however, has received less attention. For the targeted application, noninvasive physiological cues requiring minimal or no motion from the subject were desired; hence we focused on EEG, EMG, and MMG sensing modalities.

EMGs have been used extensively in HMI, in particular for control of prosthetic limbs [2], yet they require electrical conduction from the skin or through an electrode inserted invasively [3]. EMG sensors are sensitive to user body fat, interference and moisture. They are also prone to signal contamination from neighboring muscles [8]. EMG normally requires skin preparation and/or conductive gel before testing. Dry electrodes have been implemented for prosthetic hand control, but normally require expensive customization and are prone to impedance issues.

EEG has also been popular for HMI; recent studies have shown a transient response to specific applied stimuli together with a constant composition of frequencies referred to as 'brain rhythm' [9]. EEG has recently been implemented in off-line measurement for sports training [10], but remains an expensive option. Furthermore, delays in reading and data relay are unacceptable in this application. Cumbersome preparation and attachment, and latency also limit utility.

Mechanomyography is the measurement of low-frequency $(<50 \mathrm{~Hz})$ vibrations produced by muscle tissue during contraction [4]. This corresponds to the natural frequency of the muscle during twitching of fibers [5]. MMG and EMG have well-documented merits and faults. MMG is not affected by sweat, unlike EMG sensors [6]. MMG sensors are more robust to location error, need no conductive gel, are easily reusable, hence cheaper [4]. The large issue with MMG is sensitivity to interference and vibration/acoustic noise [7].

The majority of MMG research has been in disease diagnosis and rehabilitation. Clinical applications in kinesiology have studied contractile properties of motor units [11], response to ramp contractions [12], and identification of myopathies (e.g.: myotonic dystrophy) by investigating postactivation potential in bicep muscles [13] [14]. HMI using MMG has been implemented in exoskeletons, wheelchairs, and prosthetic limbs [15] [16]. Silva et al. used low frequency MMG sound signals $(5-50 \mathrm{~Hz})$ to control a prosthetic arm for 
below-elbow amputees, with the aim of creating a selfcontained system that could operate in unconstrained environments [4]. Prosthetic activation was able to achieve a delay of only $\sim 120 \mathrm{~ms}$ from intention to action, and revealed control accuracy of between $88 \%$ and $71 \%$. Antonelli et al. researched MMG signals on the rectus femoris muscle (one of four in the quadriceps) to assess its usefulness in application in lower-limb orthotic devices [14]. Other work has centered on prosthesis and orthotics, with less application in development of avocational system control.

This work investigates MMG HMI to mechanical systems for disabled and able-bodied individuals in high-performance activities, with focus on cycling. Despite many drawbacks, MMG was selected due to ease of use and sensor robustness.

\section{B. Sensor Interface system}

MMG sensors have been used to evaluate muscular phenomena in response to trigger input, passive and environmental noise. A trigger is defined as the operator purposefully contracting the left bicep muscle to cause a signal indicating, in this case, a desire to shift gears on a bicycle. MMG sensitivity to interference is addressed through the design of a filter using an inertial measurement unit to exclude vibration and human motion artefacts.

A Shimano Ultegra Di2 groupset has been fitted to a road bicycle, allowing electronic gear shifting. The Shimano Di2 groupset is an advanced electronic gear shifting system for racing bicycles. The Di2 runs on a closed source CAN system (Controller Area Network) using a bi-wire cable connected to the chip platform at the derailleur to send an 'up' or a 'down' shifting signal [17]. To maintain the original functionality, a Shimano R600 climbing switch has been added to the righthand gear shifter on the handlebars, incorporating two new switches [17].

MMG sensors have previously been made using accelerometers, piezoelectric transducers, Laser Distance Sensors (LDS) and hydrophones. The MMG implemented in this work followed the design of Woodward [19], seen in Figure 1. This sensor was fabricated based on the research of Posatski and Chou into chamber shapes [20]. To determine best noise attenuation and signal amplification, Posatski and Chou's research compared the use of cylindrical and conical chamber shapes on low frequency gain and spectral flatness [20]. Results found that a conical chamber shape offered an average rise in gain of $6.79 \pm 1.06 \mathrm{~dB} / \mathrm{Hz}$ compared to cylindrical geometries. This increase in low frequency gain is beneficial as MMG sensors focus on recording $<50 \mathrm{~Hz}$ frequencies. The conical shape allows better signal identification during use in MMG applications where limb movement is occurring due to the flatness of the wider frequency response. A Knowles' MEMS condenser microphone (SPU1410) is used as the sensor [16]. The housing of the sensor is 3D printed with the signal amplification cone sealed with a $4 \mu \mathrm{m}$ aluminum Mylar membrane [19]. In previous testing, when placed on the skin during the contraction of a lateral superficial muscle, low frequency vibrations were produced. The signal power was predominantly centered at $25 \mathrm{~Hz}$, with very little response above $100 \mathrm{~Hz}$.

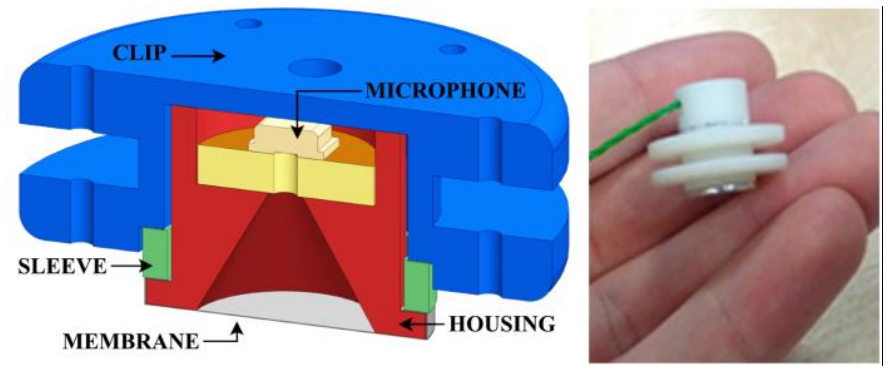

Figure 1: A rendering of the $M M G$ device used showing the microphone, housing, Mylar membrane and sleeve and the clip attached to allow for sewing on to clothing. The acoustic chamber can be seen in red [19]. A phot of the sensor is show to gauge size.

\section{HARDWARE SYSTEM DESIGN}

The shifting system consists of a biosensor data acquisition/processor attached to a user, and a trigger receiver/gear-changer system on the bicycle. The MMG signal is recorded via an Arduino Fio. Connected are an XBee radio transmitter, IMU [21] and MMG sensor to make the colloquially named MAGIE system (Mechanomyogram, Accelerometer and Gyroscopic Information Enumerator). The recorded data is sent wirelessly via the XBee radio module to a second XBee attached to a computer at a sampling frequency of $225 \mathrm{~Hz}$. Though low for MMG collection, this was adequate for this application. The system was sewn into a polyester strap designed to fit around the subject's left bicep muscle with the MMG positioned on the top of the bicep and the IMU device positioned on the outer area between bicep and tricep (see Figure 2). This is so that during tensing of the bicep muscle, the motion caused in the IMU device is minimized. The material is flexible to provide a comfortable and firm grip. MAGIE is capable of collecting, processing and outputting a response, either to a laptop for graphical visualization and post-processing or to a robotic interface for mechanical response. Post-processing in MATLAB has been used to define the signals, determine which trigger identification methods work best in practice and to apply different signal filters. Once a trigger has been identified in the processed biosignal, a trigger packet is sent to the bicycle gear-shifting system attached to the handlebars of the bicycle.
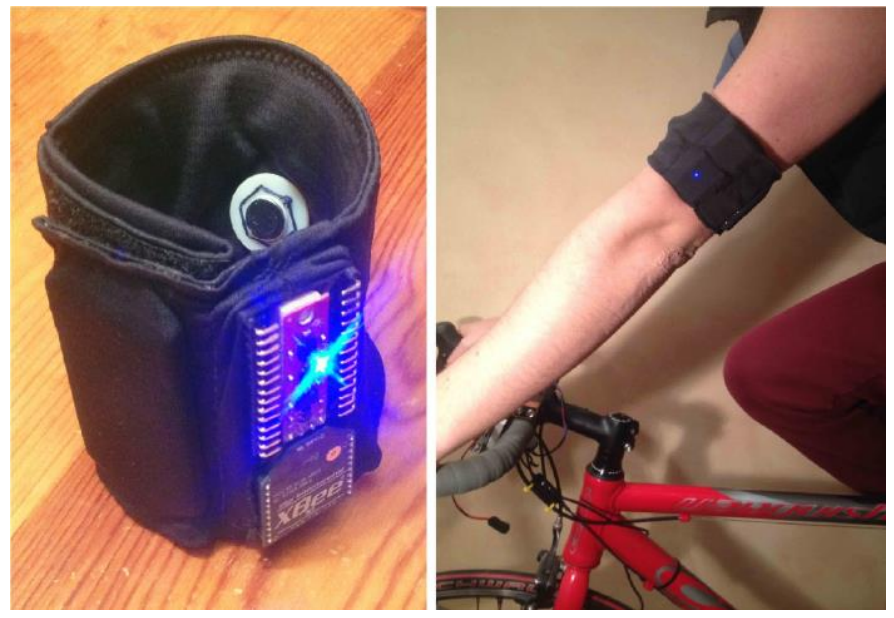

Figure 2: The colloquially named MAGIE system (Mechanomyogram, Accelerometer and Gyroscopic Information Enumerator), and position on the arm while riding a bicycle. 


\section{DATA CharaCterization}

The system has been tested in lab conditions to define the triggering signal in a minimal noise environ; then stationary cycling in lab conditions to define noise from cycling activity; and finally testing on the road to define ambient and road noise as well as noise from road hazards (e.g.: speed bumps, traffic, junctions, changing direction, etc.). Various signal filters have been applied and tested, most successfully with the Butterworth filters suggested by Fara et al. [16]. Trigger identification methods have been explored including those discussed by Rafiee et al. [22] and Staude et al. [23].

\section{A. MAGIE Signal Statistics}

Five different states have been tested to define the acceleration and MMG data characteristics. These were stationary on the lab bicycle; pedaling on the lab bicycle; cycling on the road; standing stationary and walking. Statistical data collected from each situation has been plotted in a series of box-plots in Figure 4. The results show that xand y-axis accelerations have very similar mean results when sat on a bicycle. These results differ from when stood upright or walking due to the change in arm position and can be used as a requirement for allowing a gear change: arm must be at a given angle to be sure that the operator is on the bicycle.

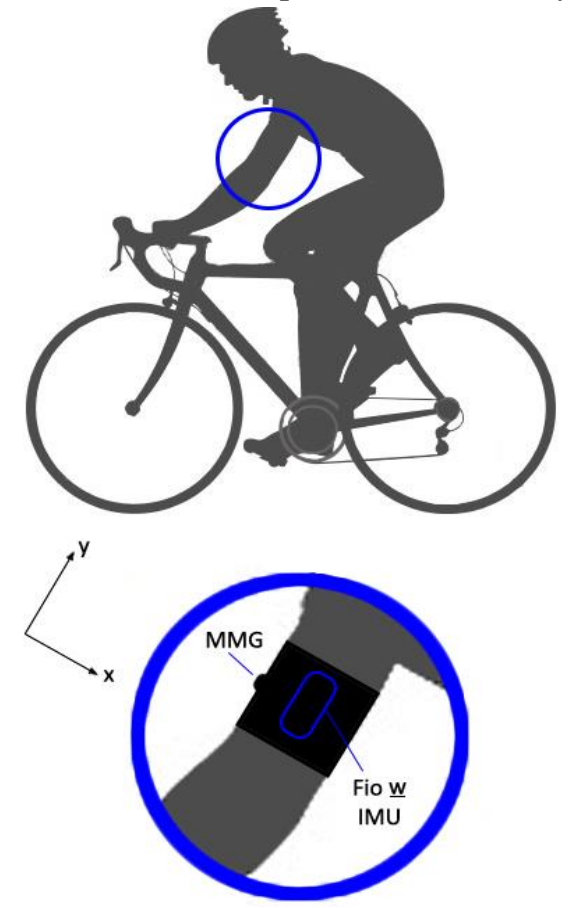

Figure 3: Positioning of the MAGIE system on the operator's left arm, with the MMG sensor placed centrally on the bicep muscle. The axes of the MAGIE system are also shown.

This requirement will stop unwanted gear changing while setting up, or while off the bicycle. The z-axis results are less defined; this is to be expected as this is the axis that changes with rotation of the arm when the shoulder and hand are stationary, and therefore is very dependent on the angle of positioning of the MAGIE system. The MMG results show a similar spread of data for walking and cycling, which suggests increased muscle activity during exercise, which is to be expected.
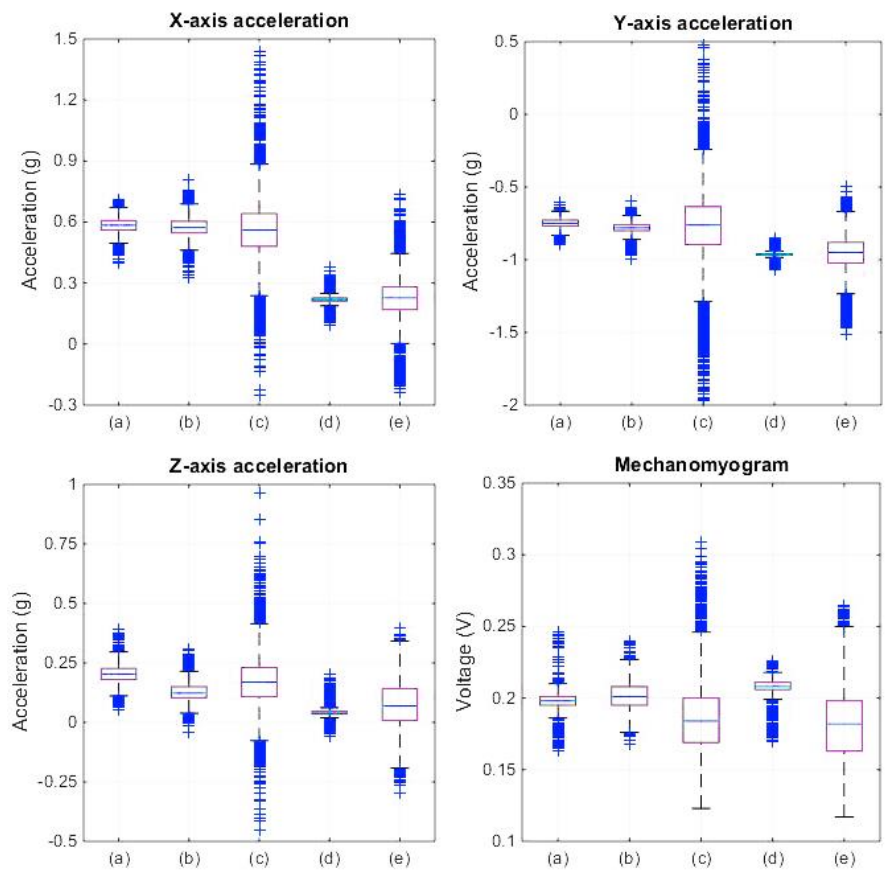

Figure 4: Box plots for x-axis, y-axis and z-axis acceleration, and $M M G$ voltage. Plots show five different testing conditions: (a) Stationary on the lab bicycle; (b) Pedaling on the lab bicycle; (c) Cycling on the road; (d) Standing stationary and (e) Walking.

\section{B. Acceleration Boundaries}

Results from 198,500 samples of data in each activity state have been collated into either upright body position (combining standing stationary and walking) or on the bicycle (combining stationary on the lab bicycle, pedaling on the lab bicycle and cycling on the road). The distribution of results for $\mathrm{x}-$ and $\mathrm{y}$-axis accelerations have been plotted in Figure $5 \& 6$.

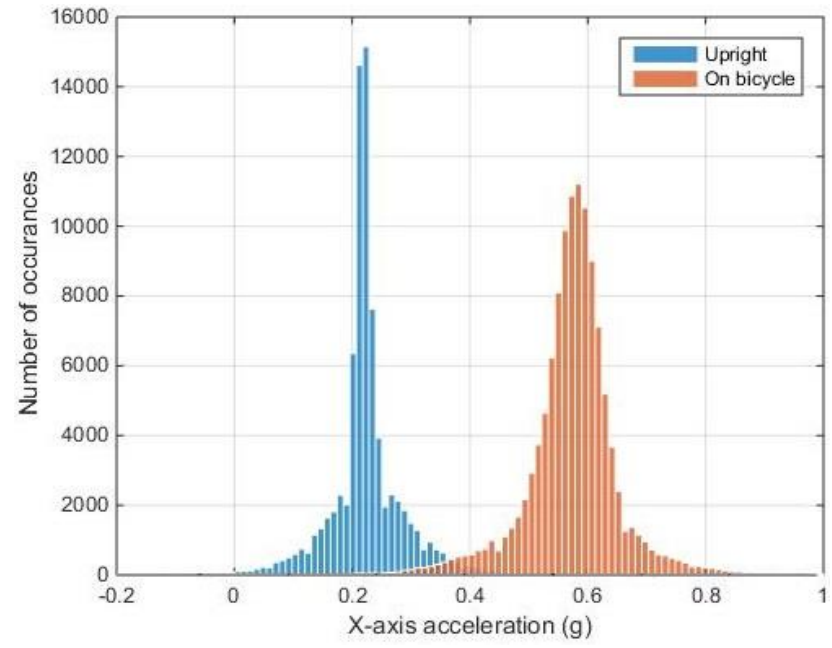

Figure 5: Comparison of $x$-axis accelerations during two body position: standing/walking upright, or sitting/pedaling on a bicycle.

The data can be seen to have normal distributions centered at different means. To define the operator's position as on the bicycle, an $x$-axis accelerometer reading of $>0.4$ and $y$-axis reading of $>-0.8$ is set. The confidence values are shown in Table 1. A combination of $\mathrm{x}$ - and $\mathrm{y}$-axis acceleration gives the best accuracy for rejecting false trigger while off the bicycle, and therefore will be used in a feedback loop. 
Table 1: Confidence results for accepting or rejecting the hypothesis that the operator is on the bicycle, rather than standing upright.

\begin{tabular}{|l|l|l|l|l|}
\hline \multirow{2}{*}{} & \multicolumn{2}{|c|}{ X-axis acceleration } & \multicolumn{2}{c|}{ Y-axis acceleration } \\
\cline { 2 - 5 } & Accept & Reject & Accept & Reject \\
\hline Correct & $98.5 \%$ & $96.9 \%$ & $99.5 \%$ & $80.9 \%$ \\
\hline Incorrect & $1.5 \%$ & $3.1 \%$ & $0.5 \%$ & $19.1 \%$ \\
\hline
\end{tabular}

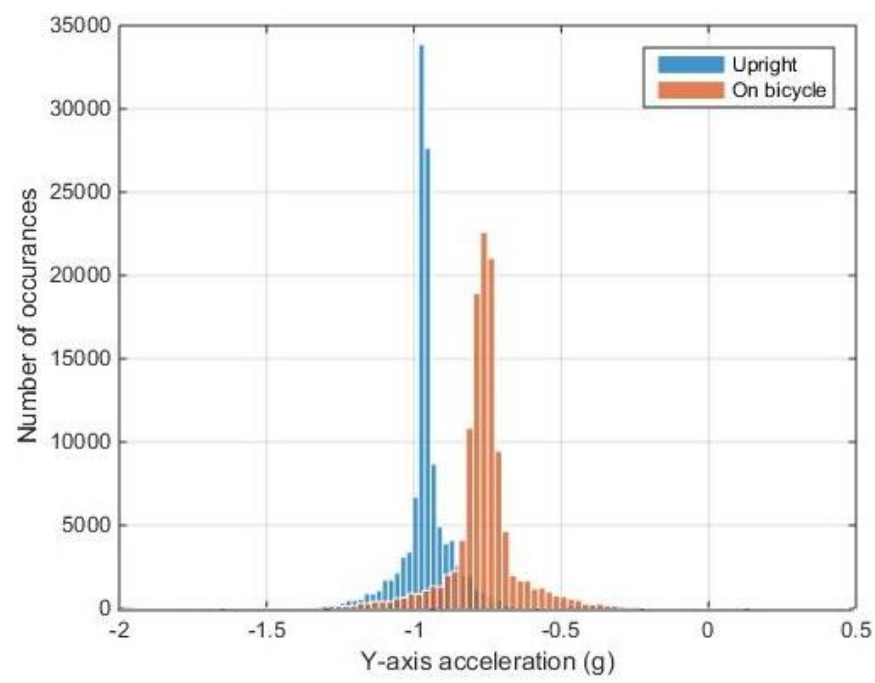

Figure 6: Comparison of y-axis accelerations during two body position: standing/walking upright, or sitting/pedaling on a bicycle.

\section{MMG Trigger Profile}

A trigger signal is defined here as a purposeful contraction of the bicep muscle within the MAGIE system. Initially a simple threshold value of $0.25 \mathrm{~V}$ was used to identify trigger signals. The identifier then waits a calibrated period of time before checking again for a threshold breach. This period was set to $2 / 9 \mathrm{sec}$, which is the maximum trigger delay.

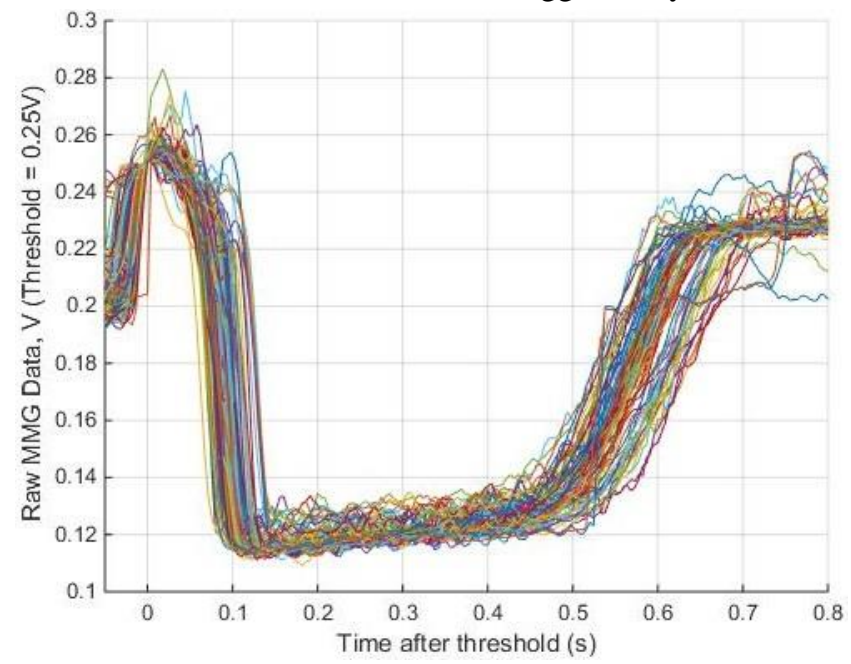

Figure 7: 95 bicep muscle triggers superimposed to show profile trend. Threshold method was used for initial identification.

This trigger identification method worked adequately during lab based testing, however was not viable for road testing where noise caused large numbers of false triggers, and so a more refined method was required. Multiple triggers were recorded while sat on the bicycle under lab conditions and superimposed in Figure 7 to assess the characteristic shape after threshold. It can be seen that the Maximum Voluntary Contraction (MVC) begins just before time zero, and the muscle is relaxing from around 0.45 seconds. The total muscle contraction profile takes approximately 0.8 seconds.

The trigger profile shows a strongly defined shape with a clear decrease in voltage after threshold, which is held for approximately $0.4 \mathrm{sec}$. The voltage distribution for a series of trigger profiles has been compared in Figure 8 to that of cycling both in lab conditions and on public roads. The histogram clearly shows a biased towards the extreme maximum and minimum regions during triggering that are differentiable from cycling in either condition.

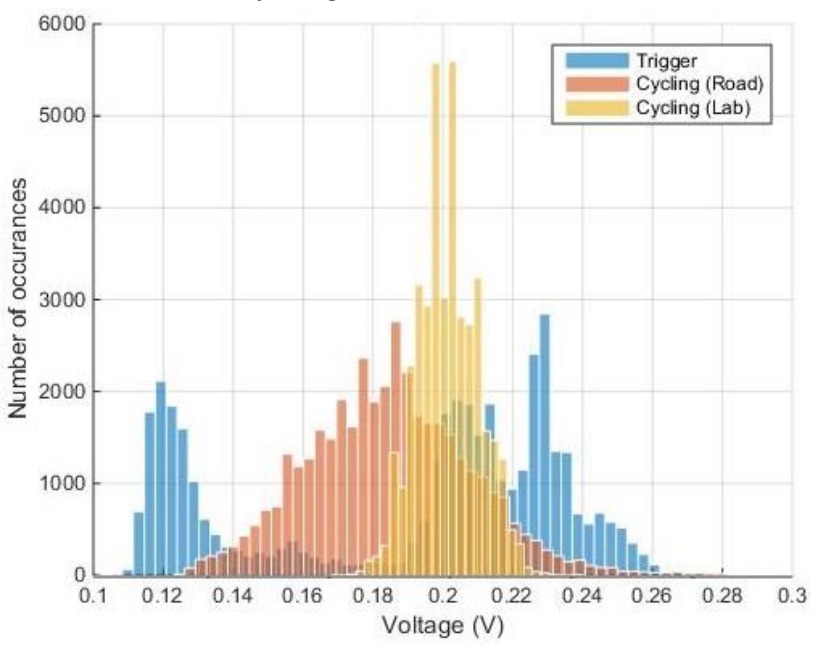

Figure 8: Histogram showing the different distributions of $M M G$ voltages for trigger profiles, cycling in the lab, and cycling on public roads.

Figure 9 shows two example triggers during lab based cycling. It can be seen that despite large fluctuations in MMG signal during non-triggering time periods, these oscillations cease during MVC and cool-down afterwards. This removal of high frequency oscillations during tensing will allow threshold methods to be applied, using both an initial upper threshold value, followed by a lower secondary threshold value $2 / 9 \mathrm{sec}$ later (during the cool-down of the muscle).

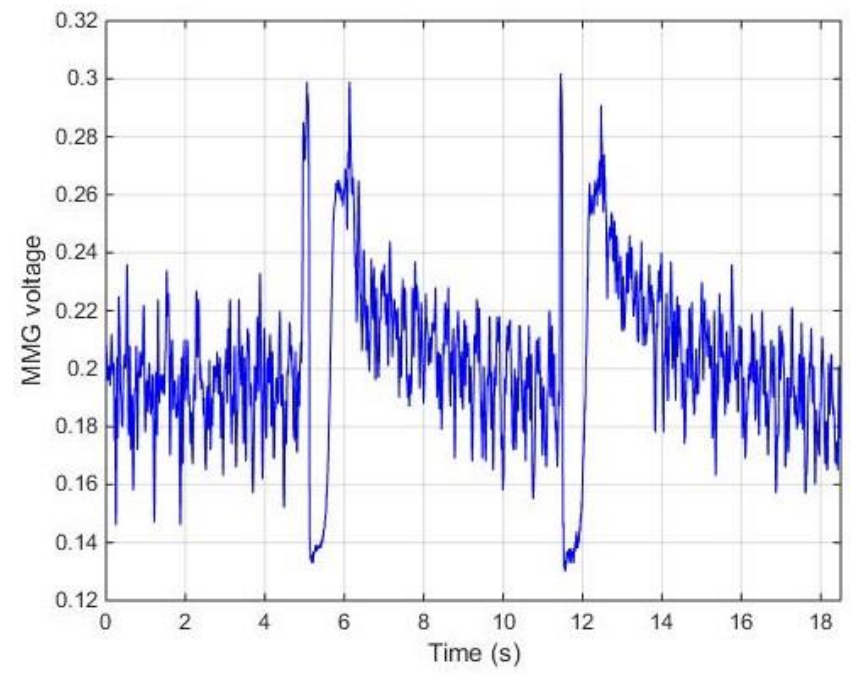

Figure 9: An extract of MMG signal data during lab based cycling. Two triggers can be observed, where the bicep muscle was contracted. 


\section{TESTING}

Statistical boundaries were applied to unfiltered data during testing. The parameters set out in Table 2 were used during lab based cycling and road cycling. Initially no filters were applied to the MMG data. Three test subjects were used in lab based testing to determine the system's use on different arm circumference and muscle size. A test was also conducted while walking for ten minutes, the results of which showed only one false trigger despite much muscle activity.

Table 2: Parameters used for trigger identification, and rejection of false triggers when not on the bicycle.

\begin{tabular}{|c|c|c|c|c|c|}
\hline \multicolumn{2}{|c|}{ MMG (V) } & \multicolumn{2}{c|}{ x-axis (g) } & \multicolumn{2}{c|}{ y-axis (g) } \\
\hline Threshold & Secondary & Upper & Lower & Upper & Lower \\
\hline 0.25 & 0.15 & 0.9 & 0.4 & 0 & -1 \\
\hline
\end{tabular}

Results of the lab based cycle testing is shown in Table 3. A 25 year old male, 30 year old male and 21 year old female were tested. The results have been displayed in a True/False, Positive/Negative table. Positive means a trigger was identified, and True means the state of trigger/no-trigger was correctly identified. A True Negative state therefore is without results as this is a null state.

Table 3: Results from cycling on a bicycle under lab conditions. Three test subjects were used of varying sex and age.

\begin{tabular}{|l|c|c|c|}
\hline & 21yo Female & 30yo Male & 25yo Male \\
\hline True Positive & $90.7 \%$ & $86.3 \%$ & $88.9 \%$ \\
\hline False Positive & $0.9 / \mathrm{min}$ & $1.2 / \mathrm{min}$ & $0.5 / \mathrm{min}$ \\
\hline False Negative & $9.3 \%$ & $13.7 \%$ & $11.1 \%$ \\
\hline
\end{tabular}

In total, 495 triggers were attempted, with 438 correctly identified. It can be seen that a successful trigger identification rate is greater than $86 \%$ for all tests conducted, with an average rate of $88.5 \%$. The average rate of falsely identified triggers was $0.87 / \mathrm{min}$. Results from road tests on public roads are shown in Table 4. 160 triggers were attempted during road testing, with 116 correctly identified.

Table 4: Results from cycling on a bicycle on public roads.

\begin{tabular}{|l|c|}
\hline & 25 yo Male \\
\hline True Positive & $72.5 \%$ \\
\hline False Positive & $3.7 / \mathrm{min}$ \\
\hline False Negative & $27.5 \%$ \\
\hline
\end{tabular}

\section{A. Frequency Profile}

The reduction in high frequency components during a muscle contraction were noted during initial testing, and could prove useful in reducing road noise in the MMG signal. By conducting a Fast Fourier Transform (FFT) on a series of bicep muscle contractions (see Figure 10) it can be seen that all important contraction information is occurring between 0 $10 \mathrm{~Hz}$, and the majority is between $2-8 \mathrm{~Hz}$. Road noise shows peaks of vibration at $<2 \mathrm{~Hz}$, and $>8 \mathrm{~Hz}$, with the majority occurring at 9 and $10 \mathrm{~Hz}$. Therefore the application of a bandpass filter was predicted to reduce road noise with minimal effect on the trigger signal amplitude.

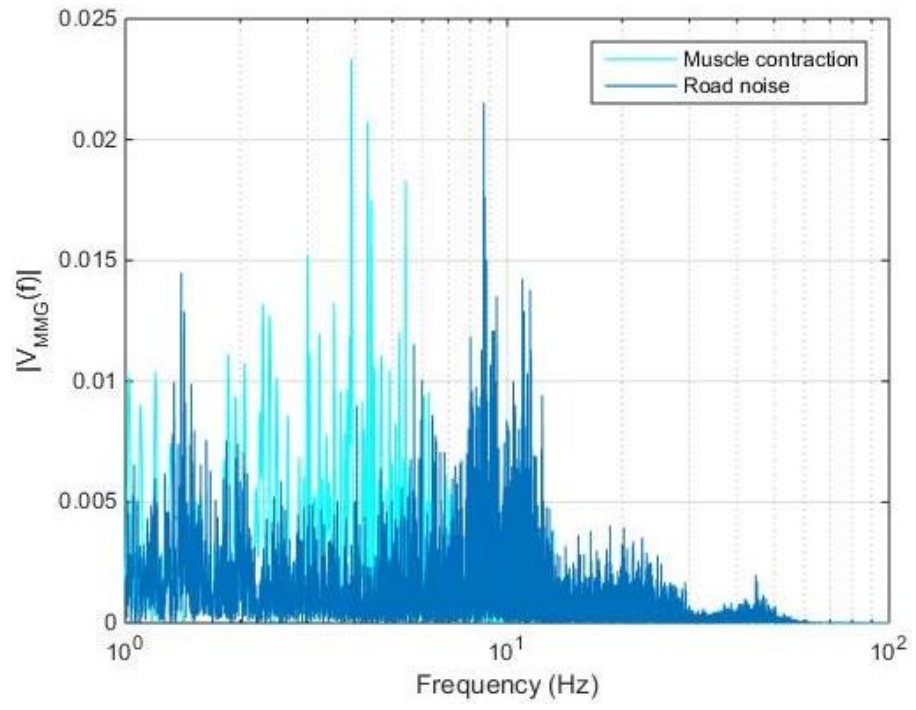

Figure 10: FFT diagram showing frequencies of MMG signal from a bicep muscle contraction, and while cycling on public roads.

Fara et al. applied a 5th order Butterworth low-pass filter to MMG data and found that it performed well, with a good compromise between high attenuation rate and flat-band pass [16]. A series of Butterworth bandpasses were applied to the MAGIE MMG signal, varying the order and upper/lower cutoff frequencies of the filter.
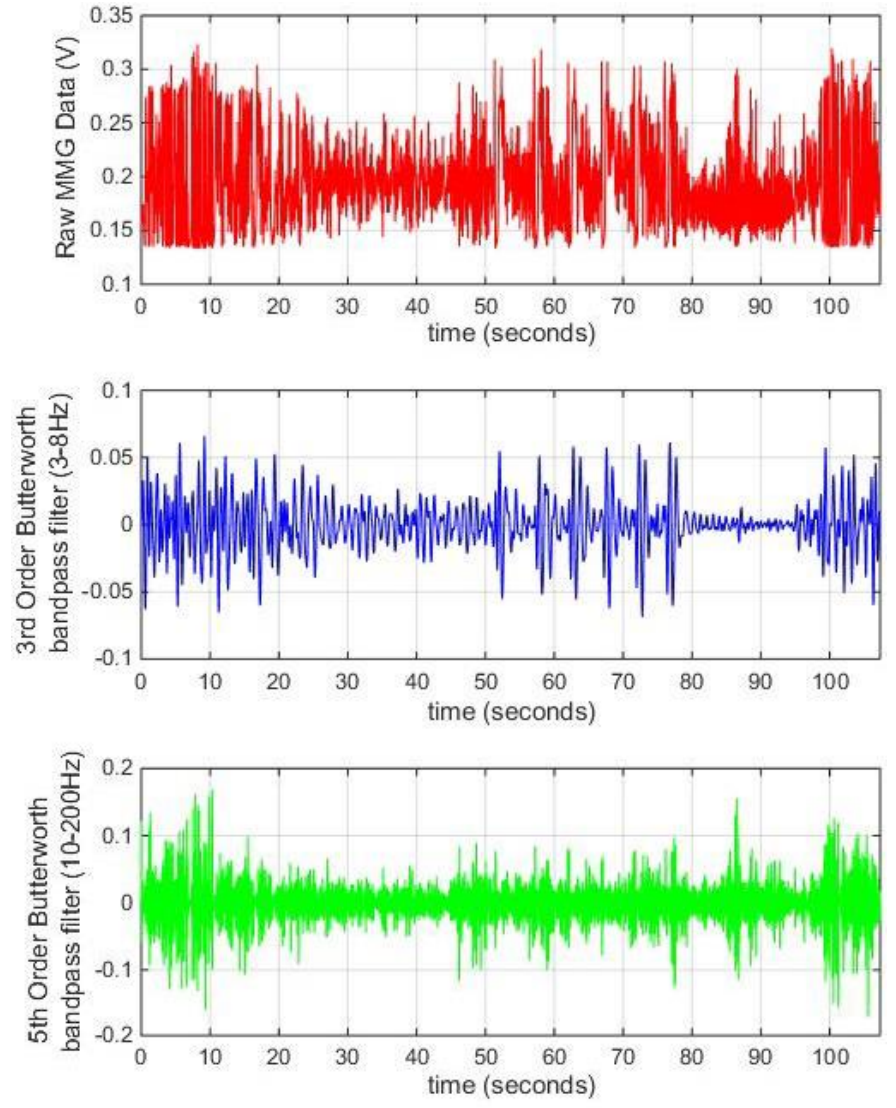

Figure 11: Results from applying Butterworth bandpass filters to MMG data collected during road cycling. Two filters have been applied: a 3rd order $3-8 \mathrm{~Hz}$, and a 5th order 10-200Hz filter. Six triggers can be seen between 50-80sec. 
The best result for reducing road noise while maintaining trigger amplitude was found using a 3rd order bandpass with normalized cut-off frequencies of $3 \mathrm{~Hz}$ (lower) and $8 \mathrm{~Hz}$ (higher). The filter was applied to some sample road test data as shown in Figure 11. The sample data shows periods of large road noise amplitude at the beginning and end, and a series of six bicep contractions between 50 and 80 seconds.

It can be seen that the $3-8 \mathrm{~Hz}$ Butterworth bandpass has greatly reduced the road noise, especially around the six triggers, while maintaining the bicep contraction signal amplitude. A 5th order $10-100 \mathrm{~Hz}$ bandpass was also applied to show the effect of cutting out all trigger data. This showed large amplitudes during large road noise, and could be used in a feedback loop to reduce the effect of road noise in the final MMG signal.

\section{CONCLUSION}

The double threshold method performed well in terms of signal identification and minimization of false positive triggers from noise. The frequency of false triggers recorded during road testing was higher than in lab testing, which is attributed to road vibrations and obstacles (such as drains, etc.) and the added requirement of the cyclist to maintain balance on the bicycle. To reduce these false positives it is suggested that a third threshold be applied at around 0.4 seconds after initial threshold. This will slow the response of the system by $0.07 \mathrm{sec}$ between muscle contraction and gear changing, however this is acceptable, as the total wait time is still below $0.5 \mathrm{sec}$.

Other trigger identification methods are available and have been explored [23] but with the application occurring in a noisy environment, road noise and arm movement are the main issue. To improve road cycling results, noise and motion cancellation must be applied. This could be in the form of a feedback loop using the $10-200 \mathrm{~Hz}$ filtered data or an acceleration based response to reject a trigger if identified during large accelerations (likely caused by changes in road condition/breaking).

\section{REFERENCES}

[1] UCI. UCI Para-Cycling Road World Championships. The Paralympian, 2(2):28-29, 2014.

[2] Mohammadreza Asghari Oskoei and Huosheng Hu. Myoelectric control systems, a survey. Biomedical Signal Processing and Control, 2(4):275 294, 2007.

[3] Gordon Robertson, Graham Caldwell, Joseph Hamill, Gary Kamen, and Saunders Whittlesey. Research Methods in Biomechanics, 2E. Human Kinetics, 2013.

[4] Jorge Silva, Winfried Heim, and Tom Chau. A self-contained, mechanomyography-driven externally powered prosthesis. Archives of Physical Medicine and Rehabilitation, 86(10):2066 - 2070, 2005.

[5] A.O. Posatskiy and T. Chau. Design and evaluation of a novel microphone-based mechanomyography sensor with cylindrical and conical acoustic chambers. Medical Engineering and Physics, 34(8):1184 - 1190, 2012.

[6] Pawel Wojtczak, Tito G. Amaral, Octavio P. Dias, Andrzej Wolczowski, and Marek Kurzynski. Hand movement recognition based on biosignal analysis. Engineering Applications of Artificial Intelligence, 22(45):608 $-615,2009$.

[7] Md Anamul Islam, Kenneth Sundaraj, R Badlishah Ahmad, and Nizam Uddin Ahamed. Mechanomyogram for muscle function assessment: a review. PloS one, 8(3):e58902, 2013.

[8] TA Kuiken, MM Lowery, and NS Stoykov. The effect of subcutaneous fat on myoelectric signal amplitude and cross-talk. Prosthetics and orthotics international, 27(1):48-54, 2003.

[9] Joo Jorge, Wietske van der Zwaag, and Patrcia Figueiredo. EEGfMRI integration for the study of human brain function. NeuroImage, 102, Part 1(0):24 - 34, 2014. Multimodal Data Fusion.

[10] Alpha-Active EEG, Head Coach System. http://www.alpha-active.com/. Accessed: 2015-20-03

[11] M. Gorelick D. McAndrew and J. M. M. Brown. Muscles within muscles: a mechanomyographic analysis of muscle segment contractile properties within human gluteus maximus. Journal of musculoskeletal research, 10(1):23 - 35, 2006.

[12] Kumi Akataki, Katsumi Mita, Makoto Watakabe, and Kunihiko Itoh. Mechanomyogram and force relationship during voluntary isometric ramp contractions of the biceps brachii muscle. European Journal of Physiology, 84(1):19 - 25, 2001.

[13] A.R. Ng, K. Arimura, K. Akataki, K. Mita, I. Higuchi, and M. Osame. Mechanomyographic determination of post-activation potentiation in myopathies. Clinical Neurophysiology, 117(1):232 - 239, 2006.

[14] Beomonte Zobel P. Antonelli M. G. and J. Giacomin. Use of $\{M M G\}$ signals for the control of powered orthotic devices: development of a rectus femoris measurement protocol. School of Engineering and Design, Brunel University, 21(1):1 - 12, 2009.

[15] Orizio C., Esposito F., Paganotti I., Marino L., Rossi B., and Veicsteinas A. Electrically-elicited surface mechanomyogram in myotonic dystrophy. Italian Journal of Neurological Science, 18(1):185 - 190, 1997.

[16] Constantinos Gavriel Salvatore Fara, Chandra Sen Vikram and A. Aldo Faisal. Robust, ultra-low-cost MMG system with brain-machineinterface applications. 6th Annual International IEEE EMBS Conference on Neural Engineering, 2013. San Diego, California.

[17] Nick Salazar, TriRig.com. Shimano Ultegra Di2 Shifters for Time Trials and Triathlon. http://www.tririg.com/articles.php?id=2012 08 Shimano Ultegra Di2 TT. Accessed: 2014-11-12.

[18] Matthew Tan Teik Hoe. Rapid bicycle gear switching based on physiological cues. Individual Project Final Report, Imperial College London, UK. 2014.

[19] R. Woodward, S. Shefelbine, R. Vaidyanathan, "Pervasive motion tracking and muscle activty monitor," IEEE 27th International Symposium on Computer-Based Medical Systems (CMBS), pp 421-26, May 2014

[20] A.O. Posatskiy and T. Chau. Design and evaluation of a novel microphone-based mechanomyography sensor with cylindrical and conical acoustic chambers. Medical Engineering and Physics, 34(8): $1184-1190,2012$.

[21] Spark Fun. 9 degrees of freedom - sensor stick. http://proto-pic.co.uk/9degrees-of-freedom-sensor-stick/. Accessed: 2015-01-15.

[22] J. Rafiee, M.A. Rafiee, F. Yavari, and M.P. Schoen. Feature extraction of forearm $\{\mathrm{EMG}\}$ signals for prosthetics. Expert Systems with Applications, 38(4):4058 - 4067, 2011.

[23] Gerhard Staude, Claus Flachenecker, Martin Daumer, and Werner Wolf. Onset detection in surface electromyographic signals: A systematic comparison of methods. EURASIP Journal on Applied Signal Processing, 2(2):67 - 81, 2001. 